PROCEEDINGS OF THE

AMERICAN MATHEMATICAL SOCIETY

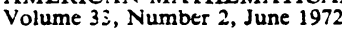

\title{
PURE STATES WITH THE RESTRICTION PROPERTY
}

\author{
BRUCE A. BARNES ${ }^{1}$
}

\begin{abstract}
Conditions are given which impiy that a pure state of a $B^{*}$-algebra $A$ restricts to a pure state of some maximal commutative *-subalgebra of $A$.
\end{abstract}

1. Introduction. A pure state $\rho$ of a $B^{*}$-algebra $A$ has the restriction property if there exists a maximal commutative *-subalgebra $C$ of $A$ such that the restriction of $\rho$ to $C$ is a pure state of $C$ (i.e. $\rho$ is a nonzero multiplicative linear functional on $C$ ). The work of $\mathrm{R}$. Kadison and I. Singer in [4] raises the question of whether or not each pure state of a $B^{*}$-algebra has the restriction property. This question was answered by J. Aarnes and $\mathrm{R}$. Kadison for a special class of $B^{*}$-algebras $A$. They prove that when $A$ is separable and has an identity, then each pure state of $A$ has the restriction property [1, Theorem 2]. Again in the case when $A$ is separable, C. Akemann in [2] removed the requirement that $A$ have an identity and made other improvements in the result of Aarnes and Kadison (including a proof that in this case a pure state $\rho$ of $A$ is the urique extension of a pure state of some maximal commutative *-subalgebra of $A$ ). However, the general question remains open.

In this note we give several new conditions on a pure state $\rho$ of a $B^{*}$ algebra which inply that $\rho$ has the restriction property. $A$ is a $B^{*}$-algebra throughout. Let $a \rightarrow \pi(a)$ be a ${ }^{*}$-representation of $A$ on a Hilbert space $\mathscr{H}$. A positive functional $\rho$ is represented by $\pi$ if there is $\xi \in \mathscr{H},\|\xi\|=1$, such that $\rho(a)=(\pi(a) \xi, \xi)$ for all $a \in A$. A pure state of $A$ is always represented by some irreducible *-representation of $A$; see $[3, \mathrm{pp} .32,33,37]$ for details. Now let $\rho$ be a pure state of $A$ which is represented by an irreducible *-representation $\pi$ of $A$ on a Hilbert space $\mathscr{H}$. We prove that if either $\mathscr{H}$ is separable or $\pi(A)$ contains $\mathscr{F}(\mathscr{H})$, the algebra of bounded operators on $\mathscr{H}$ with finite dimensional range, then $\rho$ has the restriction property. The proofs of these results are indebted to the ideas of Aarnes and Kadison in [1].

2. The results. Let be $\mathscr{H}$ a Hilbert space. $\mathscr{B}(\mathscr{H})$ is the algebra of bounded operators on $\mathscr{H}$. When $\mathscr{K}$ is a subspace of $\mathscr{H}$ and $B$ is a

Received by the editors September 15, 1971.

AMS 1970 subject classifications. Primary 46L05.

Key words and phrases. $B^{*}$-algebra, pure state, naximal commutative *-subalgebra. ${ }^{1}$ This research was supported by National Science Foundation Grant GP-28250.

(C) American Mathematical Society 1972 
nonempty subset of $\mathscr{B}(\mathscr{H})$, then $B \mathscr{K}$ is the linear span of the vectors $\{T \psi \mid T \in B, \psi \in \mathscr{K}\} .[B \mathscr{K}]$ is the closure of $B \mathscr{K}$ in $\mathscr{H}$. When $T \in \mathscr{B}(\mathscr{H})$, then $\mathscr{N}(T)$ is the null space of $T$ and $\mathscr{R}(T)$ is the range of $T$.

LEMMA. Let $\mathscr{H}$ be a separable Hilbert space and assume that $A$ is a closed *-subalgebra of $\mathscr{B}(\mathscr{H})$ such that $[A \mathscr{H}]=\mathscr{H}$. Then there exists $T \in A, T \geqq 0$, such that $\mathfrak{N}(T)=0$.

Proof. Since $[A \mathscr{H}]=\mathscr{H}$, then given any $\psi \in \mathscr{H}, \psi \neq 0$, there exists $S \in A$ such that $S \psi \neq 0$. Then also $S^{*} S \psi \neq 0$. Therefore for each $\psi \in \mathscr{H}$, $\psi \neq 0$, we can choose $T_{\psi} \in A$ such that $T_{\psi} \geqq 0$ and $T_{\psi}(\psi) \neq 0$. Let $U_{\psi}=$ $\left\{\xi \in \mathscr{H} \mid T_{\psi}(\xi) \neq 0\right\}$. The collection $\left\{U_{\psi} \mid \psi \in \mathscr{H}, \psi \neq 0\right\}$ is an open cover for $\mathscr{H} \backslash\{0\} . \mathscr{H} \backslash\{0\}$ being a separable metric space is Lindelöf (every open cover has a countable subcover). It follows that there exists a sequence $\left\{T_{n}\right\} \subset A$ such that $T_{n} \geqq 0$ and $\bigcap_{n=1}^{+\infty} \mathcal{N}\left(T_{n}\right)=0$. Let $a_{n}=\left(2^{n}\left\|T_{n}\right\|\right)^{-1}$, and set $T=\sum_{n=1}^{+\infty} a_{n} T_{n}$. If $T \psi=0$, then $\sum_{n=1}^{+\infty} a_{n}\left(T_{n} \psi, \psi\right)=0$. Therefore $\left(T_{n} \psi, \psi\right)=$ 0 for each $n$. But then $T_{n}^{1 / 2} \psi=0$, which implies $T_{n} \psi=0$ for each $n$. Therefore $\psi=0$.

When $D$ is a nonempty subset of $A$, we let

$$
\mathscr{C}(D)=\{a \in A \mid a d=d a \text { for all } d \in D\} .
$$

If $D$ is selfadjoint, then $\mathscr{C}(D)$ is a closed *-subalgebra of $A$.

THEOREM 1. Let $a \rightarrow \pi(a)$ be an irreducible *-representation of $A$ on $a$ separable Hilbert space $\mathscr{H}$. If $\rho$ is a positive functional represented by $\pi$, then $\rho$ has the restriction property.

Proof. There exists $\xi \in \mathscr{H},\|\xi\|=1$, such that $\rho(a)=(\pi(a) \xi, \xi)$ for all $a \in A$. Let $K=\left\{a \in A \mid \rho\left(a^{*} a\right)=0\right\}=\{a \in A \mid \pi(a) \xi=0\}$. Set $A_{0}=K \cap K^{*}$ and $\mathscr{H}_{0}=\{\xi\}^{\perp}$. Given $a \in A_{0}$ and $\psi \in \mathscr{H}$, we have $(\pi(a) \psi, \xi)=\left(\psi, \pi\left(a^{*}\right) \xi\right)=0$. Therefore $\pi\left(A_{0}\right) \mathscr{H} \subset \mathscr{H}_{0}$. Let $E$ be the orthogonal projection of $\mathscr{H}$ onto $\mathscr{H}_{0}$. Then

$$
E \pi(a)=\pi(a) \text { for all } a \in A_{0} .
$$

By [3, Corollaire (2.8.4)], $\pi(A)$ acts strictly irreducibly on $\mathscr{H}$. Therefore there exists $v \in A$ such that $\pi(v) \xi=\xi$. Since $\pi\left(v+v^{*}-v^{*} v\right) \xi=\xi$, we may assume that $v=v^{*}$. Set $u=2 v-v^{2}$. Then $I-\pi(u)=(I-\pi(v))^{2} \geqq 0$ where $I$ is the identity operator on $\mathscr{H}$. If $\psi \in \mathscr{H},((I-\pi(u)) \psi, \xi)=\left(\psi,(I-\pi(v))^{2} \xi\right)=0$. Therefore

$$
E(I-\pi(u))=I-\pi(u) .
$$

Given $\psi \in \mathscr{H}_{0}$, the transitivity theorem [3, Théorème (2.8.3)] implies that there exists $a \in A$ such that $a=a^{*}, \pi(a) \xi=0$, and $\pi(a) \psi=\psi$. Then $a \in A_{0}$, and this proves that $\pi\left(A_{0}\right) \mathscr{H}_{0}=\mathscr{H}_{0}$. By the Lemma there exists $w \in A_{0}, w \geqq 0$, 
such that $\mathscr{A}^{\prime}(\pi(w)) \cap \mathscr{H}_{0}=0$. Set $S=I-\pi(u)+\pi(w)$. Since $I-\pi(u) \geqq 0$, then $\mathcal{A}^{\prime \prime}(S) \cap \mathscr{K}_{0}=0$. Let $y=u-w$, and choose $C_{0}$ a maximal commutative *-subalgebra of $\mathscr{C}(y) \cap A_{0}$. Let $C$ be the closed commutative *-subalgebra of $A$ generated by $y$ and $C_{\mathrm{c}}$. We prove that $C$ is a maximal commutative *-subalgebra of $A$. Assume that $b=b *$ and $b \in \mathscr{C}(C)$. Let $b_{0}=b-\rho(b) y$. Then $b_{0}=b_{0}^{*}$ and $b_{0} \in \mathscr{C}(C)$. Using (1) and (2) we have $E S=E(I-\pi(u)+\pi(w))=$ $I-\pi(u)+\pi(w)=S$. Also $\pi\left(b_{0}\right) S=S \pi\left(b_{0}\right)$. Then

$$
\left(E \pi\left(b_{0}\right)-\pi\left(b_{0}\right) E\right) S=S \pi\left(b_{0}\right)-\pi\left(b_{0}\right) S=0 .
$$

Since $\mathscr{N}(S) \cap \mathscr{H}_{0}=0$ and $S=S^{*}$, then $(\mathscr{R}(S))^{-}=\mathscr{H}_{0}=\mathscr{R}(E)$. Therefore $\left(E \pi\left(b_{0}\right)-\pi\left(b_{0}\right) E\right) E=0$. It follows that $\pi\left(b_{0}\right) E=E \pi\left(b_{0}\right)$. Then there exists a scalar $\lambda$ such that $\pi\left(b_{0}\right) \xi=\lambda \xi$. Note that $\rho(y)=\rho(u-w)=$ $\rho(u)=\rho\left(2 v-v^{2}\right)=\left(\pi\left(2 v-v^{2}\right) \xi, \xi\right)=1$. Therefore $\lambda=\left(\pi\left(b_{0}\right) \xi, \xi\right)=\rho\left(b_{0}\right)=$ $\rho(b-\rho(b) y)=0$. Then $b_{0} \in \mathscr{C}(y) \cap A_{0}$, and it follows that $b_{0} \in C_{0}$. But then $b \in C$. This proves that $C$ is a maximal commutative *-subalgebra of $A$.

$\rho$ is nonzero on $C$ since $\rho(y)=1$. It remains to be shown that $\rho$ is multiplicative on $C$. Given $a \in C_{0}$, then $y a \in \mathscr{C}(y)$. Also $\pi(y a) \xi=\pi(y) \pi(a) \xi=0$ and similarly $\pi\left(a^{*} y\right) \xi=0$. Therefore $y a$ and $(y a)^{*}$ are in $\mathscr{C}(y) \cap A_{0}$. Thus $y a \in C_{0}$. Furthermore $\pi(y) \xi=(\pi(u)-\pi(w)) \xi=\pi(u) \xi=\xi$. Thus $\pi\left(y^{n}-y\right) \xi=0$ for any positive integer $n$. Then $y^{n}-y \in \mathscr{C}(y) \cap A_{0}$, and therefore $y^{n}-y \in C_{0}$ for each positive integer $n$. It follows that every element of $C$ has the form $\lambda y+a$ for some scalar $\lambda$ and some $a \in C_{0}$. Then given $\lambda, \mu$ scalars and $a$, $b \in C_{0}$,

$$
\rho((\lambda y+a)(\mu y+b))=\lambda \mu=\rho(\lambda y+a) \rho(\mu y+b) .
$$

This completes the proof of the theorem.

In the case where $A$ has an identity, the proof of Theorem 1 can be considerably simplified.

THEOREM 2. Let $a \rightarrow \pi(a)$ be $a *$-representation of $A$ on a Hilbert space $\mathscr{H}$ with the property that $\mathscr{F}(\mathscr{H}) \subset \pi(A)$. If $\rho$ is a positive functional represented by $\pi$, then $\rho$ has the restriction property.

Proof. Assume that $\rho(a)=(\pi(a) \xi, \xi)$ for all $a \in A$, where $\xi \in \mathscr{H}$, $\|\xi\|=1$. Let $K=\{a \in A \mid \pi(a) \xi=0\}$, and set $A_{0}=K \cap K^{*}$. Let $E$ be the orthogonal projection with one dimensional range containing $\xi$. By hypothesis there exists $e \in A, e=e^{*}$. such that $\pi(e)=E$. Choose $C_{0}$ a maximal commutative ${ }^{*}$-subalgebra of $\mathscr{C}(e) \cap A_{0}$. Let $C$ be the closed commutative *-subalgebra of $A$ generated by $e$ and $C_{0}$. Assume that $b=b^{*} \in \mathscr{C}(C)$. Sei $b_{0}=b-\rho(b) e$. Note that $\rho(\varepsilon)=(E \xi, \xi)=1$, so that $\rho\left(b_{0}\right)=0$. Then $\pi\left(b_{0}\right) E=E \pi\left(\dot{b}_{0}\right)$. Therefore there exists a scalar $\lambda$ such that $\pi\left(b_{0}\right) \xi=\lambda . \xi$. Then $\lambda=\left(\pi\left(b_{0}\right) \xi, \xi\right)=\rho\left(b_{0}\right)=0$. It follows that $b_{0} \in \mathscr{C}(e) \cap$ $A_{0}$, so that by the definition of $C_{0}, b_{0} \in C_{0}$. Then $b \in C$. This proves that $C$ is 
a maximal commutative *-subalgebra of $A$. The proof that $\rho$ is a nonzero multiplicative functional on $C$ proceeds as in the last paragraph of the proof of Theorem 1 with $e$ in place of $y$.

When $A$ is a GCR algebra (postliminaire) and $a \rightarrow \pi(a)$ is an irreducible *-representation of $A$ on a Hilbert space $\mathscr{H}$, then it is well known that $\mathscr{F}(\mathscr{H}) \subset \pi(A)$; see [3, Théorème (4.3.7)]. Therefore we have as a corollary of Theorem 2:

Corollary. A pure state of a GCR algebra $A$ has the restriction property.

\section{REFERENCES}

1. J. F. Aarnes and R. V. Kadison, Pure states and approximate identities, Proc. Amer. Math. Soc. 21 (1969), 749-752. MR 39 \#1980.

2. C. A. Akemann, Approximate units and maximal Abelian $C^{*}$-subalgebras, Pacific J. Math. 33 (1970), 543-550. MR 41 \#9001.

3. J. Dixmier, Les $C^{*}$-algèbres et leurs représentations, Cahiers Scientifiques, fasc. 29, Gauthier-Villars, Paris, 1964. MR 30 \#1404.

4. R. V. Kadison and I. M. Singer, Extensions of pure states, Amer. J. Math. 81 (1959), 383-400. MR 23 \#A1243.

Department of Mathematics, University of Oregon, Eugene, Oregon 97403 